\title{
Generalized lattice Boltzmann model for frosting
}

\author{
Timan Lei, ${ }^{1}$ Kai H. Luo, ${ }^{1, *}$ and Duan $\mathrm{Wu}^{2}$ \\ ${ }^{1}$ Department of Mechanical Engineering, University College London, Torrington Place, London WC1E 7JE, United Kingdom \\ ${ }^{2}$ Future Cooling Technology Ltd, Oldwood Place, Livingston EH54 6UX, United Kingdom
}

(Received 7 October 2018; revised manuscript received 18 February 2019; published 1 May 2019)

\begin{abstract}
Frosting is a multiscale and multiphysics problem, which presents a significant challenge for numerical methods. In this study, a generalized lattice Boltzmann (LB) model is developed to simulate the frosting of humid air at representative elementary volume scale. In this model, three LB equations are introduced to describe the evolution of distribution functions for velocity, temperature, and humidity (i.e., mass fraction of water vapor in the humid air) fields, respectively. The frost layer is regarded as a porous medium, while the humid air is treated as a plain one. This unified LB model can be applied to describe the phase change and transport processes in these two subdomains seamlessly. Through the Chapman-Enskog analysis, the macroscopic equations for the frosting process can be recovered from the present LB model. Benchmark problems in conduction solidification, convection melting and frosting are simulated, and the numerical results match well with analytical or experimental solutions. Finally, this model is applied to simulate frost formation between two parallel plates, and the influences of air velocity, humidity, temperature, and cold wall temperature are evaluated.
\end{abstract}

DOI: 10.1103/PhysRevE.99.053301

\section{INTRODUCTION}

Frosting occurs once moist air comes into contact with a cold plate whose temperature is below $0^{\circ} \mathrm{C}$. This phenomenon appears in various natural processes and engineering applications, including cryogenics, aeronautics, refrigeration, and heat exchangers [1]. In these fields, frost accumulation is highly undesirable, since it can result in thermal performance degradation, flow passage block, and additional pressure drop [2-4]. Thus, for the sake of optimizing application performance under frosting conditions, it is necessary to investigate the mechanisms behind frosting and predict the onset and development of the frosting process.

The frosting process involves unsteady fluid flow, heat and mass transfer, phase change, and variable porous media simultaneously. For this kind of complex problem, analytical solutions are difficult to obtain. Mathematical models and related numerical simulations turn out to be effective approaches. Over the past several decades, a variety of frosting models have been reported, in which the physical domain is divided into two subdomains, namely, the plain humid air region and the porous frost layer. Since the structure of the frost layer is complex at pore scale, fluid transport is usually modeled at representative elementary volume (REV) scale using the volume-averaging techniques. The much larger REV scale can both hold scale characteristics of porous flows and ignore pore-scale details [5]. Depending on the governing equations for frosting properties in these two subdomains, existing models can be divided into three groups.

In the first group, governing equations are only established for the frost layer, while heat and mass transfer in the

*Corresponding author: k.luo@ucl.ac.uk humid air region are calculated using empirical correlations [6-10]. It is emphasized that all these models are one dimensional and need experimental determination for correlations. Therefore, the second group has been developed to include two sets of governing equations for the frost layer and the humid air region, respectively. Additional air-frost interface conditions are built to connect these two subdomains. For instance, Luer et al. [11] and Lee et al. [12] presented both transient and quasi-steady-state models. Lenic et al. $[13,14]$ then formulated a model based on supersaturation assumption, and Armengol et al. [15] further developed one with a twodimensional (2D) frost growth rate. These models can study the frosting process in 2D configurations, but they completely ignore the convection effects within the frost layer.

More recently, the third group solves governing equations for air and ice phases in the whole frosting domain and describes the mass transfer rate from air to ice phase by different schemes. For instance, Cui et al. $[16,17]$ used the nucleation theory to calculate the mass transfer term; Kim et al. [18] assumed the mass transfer rate was driven by water vapor concentration gradients; Wu et al. [19,20] attributed it to the difference between water vapor pressure and saturation pressure. Generally, these models can address the deficiencies in the first two groups. But additional momentum and energy transfer conditions between two phases need to be realized. It can be concluded that, although models for the frosting process are abundant, a generalized one that treats both subdomains and both phases together is still missing.

Existing literatures indicate that, based on the volumeaveraging procedure, governing equations at REV scale can be derived to describe the fluid transport in heterogenous media with more than one typical pore size $[5,21]$. The more comprehensive one was introduced by Faghri et al. [21], including the continuity, momentum, energy, and species equations. Due 
to the complexity of the REV-scale equations, much effort has been made to develop efficient numerical solvers. Based on conventional computational fluid dynamics models, such as the finite-volume method [22,23], the finite-difference method [24], and the finite-element method [25,26], numerical studies have been carried out to investigate the fluid transport and phase change problems in porous media at REV scale. Different from these continuum models, the particle-based lattice Boltzmann (LB) method has been developed over the past three decades. The LB method has become an attractive alternative to conventional solvers for porous transport with and without phase changes [27-30], which attributes to its simple implementation, high parallelism, and ability to handle complex physics and boundary conditions. For example, Guo et al. [5] proposed an LB model for incompressible flow in porous media. This model is reported to be applicable for transient flow in a medium with both constant and variable porosities. Subsequently, Guo et al. [31] extended their model to involve convective heat transfer by introducing another LB equation for the temperature field. On the basis of this, some efforts have been made to further include the liquid-solid phase change process in porous media. For instance, Gao et al. $[32,33]$ developed a LB model for the phase change process in porous media under thermal equilibrium condition, and Liu et al. [34,35] and Wu et al. [36] proposed multiple-relaxationtime LB models to improve the numerical stability.

In summary, REV-scale governing equations and related LB models have been proven to be efficient in describing flow and phase change processes in media with variable porosities. In terms of their applications in the frosting process, however, two deficiencies should be pointed out. First, existing models are limited to phase change problems between two phases with the same density, and mass sources are not involved in their fluid dynamical equations. During the frosting process, however, the humid air and ice phases have significantly different densities. A mass source describing the mass transfer between humid air and ice phases is thus required to conserve mass locally [21]. Second, previous models calculate the variable porosity depending on the temperature field merely. But the variable porosity of frost layer is determined by the amount of mass transferred from humid air to ice phase, and this mass transfer rate is controlled by both temperature and humidity fields [16]. Inclusion of the humidity field is therefore necessary in the study of the frosting process.

To fill these gaps, in this work, a set of generalized governing equations are developed to describe the multiphysics processes in both the frost layer and the humid air part in a unified fashion. The mass source is considered, and the newly introduced humidity field affects the porosity of frost layer together with the temperature field. The corresponding generalized LB model is also developed, with the velocity, temperature, and humidity fields being described by three LB equations separately. Through the Chapmann-Enskog expansion, the governing equations for the frosting process are derived from the present LB model. The rest of this paper is organized as follows. In Secs. II-IV, the governing equations and the related LB models are first introduced and validated; then the frosting process in a parallel channel is simulated with different parameters in Sec. V; and, finally, a summary of the present work is provided in Sec. VI.

\section{GENERALIZED GOVERNING EQUATIONS}

Without loss of generality, frosting of humid air over a cold plate is considered in a $2 \mathrm{D}$ domain with length $l_{x}$ and width $l_{y}$. In this system, the steady cold plate is maintained at temperature $T_{w}$, which is below the freezing point. The incompressible humid air with temperature $T_{a}$ and humidity $W_{v, 0}$ flows on the cold wall with velocity $\boldsymbol{u}=\left(u_{0}, 0\right)$. The frosting process takes place as soon as the water vapor in humid air and cold plate are put into contact to yield ice droplets. During frosting, the frost layer is regarded as a porous medium with a variable porosity $\epsilon$, and this fluidsaturated medium consists of ice matrix and air gaps. Due to the complex porous structure, flow in porous frost layer is usually modeled at REV scale by using the volume-averaging procedure. In this way, the elementary control volume $V$ is introduced, where humid air and ice matrix coexist, and their volume fractions $f$ are defined as [21]

$$
f_{l}=\epsilon=\frac{V_{l}}{V}, \quad f_{s}=1-\epsilon .
$$

The subscripts $s$ and $l$ refer to the properties of ice phase and humid air, respectively. Then the volume-averaged physical quantity $\langle\psi\rangle$ can be calculated as [21]

$$
\langle\psi\rangle=\psi_{s} f_{s}+\psi_{l} \epsilon
$$

where $\psi$ is the corresponding physical quantity. It is obvious that $\epsilon=1$ for the plain humid air region and $0<\epsilon<1$ for the porous frost layer.

Based on this volume-average technique and the local thermal equilibrium assumption, the continuity equation, the generalized non-Darcy (or Darcy-Brinkman-Forchheimer) equation, and the energy and species conservation equations during the frosting process at REV scale can be developed as [21]

$$
\begin{gathered}
\rho_{l} \frac{\partial \epsilon}{\partial t}+\rho_{l} \nabla \cdot \boldsymbol{u}=-\dot{m} \\
\rho_{l} \frac{\partial \boldsymbol{u}}{\partial t}+\rho_{l} \boldsymbol{u} \cdot \nabla\left(\frac{\boldsymbol{u}}{\epsilon}\right)=-\nabla(\epsilon p)+\rho_{l} v_{e} \nabla^{2} \boldsymbol{u}+\rho_{l} \boldsymbol{F}+\boldsymbol{M} \\
\sigma \frac{\partial T}{\partial t}+\boldsymbol{u} \cdot \nabla T=\alpha_{e} \nabla^{2} T-\frac{L_{a}}{c_{p, l}} \frac{\partial \epsilon}{\partial t} \\
\epsilon \frac{\partial W_{v}}{\partial t}+\boldsymbol{u} \cdot \nabla W_{v}=D_{e} \nabla^{2} W_{v}-\frac{\dot{m}}{\rho_{l}}
\end{gathered}
$$

where $\boldsymbol{u}, p, T$, and $W_{v}$ are the volume-averaged velocity, pressure, temperature, and humidity, respectively. $\dot{m}$ is the mass transfer rate from water vapor to ice phase, $\rho_{l}$ is the humid air density, and $L_{a}$ denotes the latent heat. The parameters $v_{e}$ and $D_{e}$ are the effective values of viscosity and diffusion coefficients, respectively. The heat capacity ratio $\sigma$ is calculated by [21]

$$
\sigma=\frac{\rho_{l} c_{p, l} \epsilon+\rho_{s} c_{p, s} f_{s}}{\rho_{l} c_{p, l}},
$$

where $c_{p}$ is the specific heat at constant pressure. The effective thermal diffusivity $\alpha_{e}$ is defined as [21]

$$
\alpha_{e}=\frac{\lambda_{e}}{\rho_{l} c_{p, l}},
$$

with $\lambda_{e}$ representing the effective thermal conductivity. 
The last two terms in Eq. (4) symbolize the momentum transfer rate $\boldsymbol{M}$ in the frosting process, and the total body force $\boldsymbol{F}$, which is also called the Darcy-Brinkman-Forchheimer force. It is calculated as [5]

$$
\boldsymbol{F}=-\frac{\epsilon v_{l}}{K} \boldsymbol{u}-\frac{\epsilon F_{\epsilon}}{\sqrt{K}}|\boldsymbol{u}| \boldsymbol{u}+\epsilon \boldsymbol{G},
$$

where $v_{l}$ is the viscosity of humid air and $\boldsymbol{G}$ is the external force. The first and the second terms on the right-hand side of Eq. (9) are the linear (Darcy) and nonlinear (Forchheimer) drags due to the presence of the porous frost layer. The geometric function $F_{\epsilon}$ and the permeability $K$ can be expressed as $[37,38]$

$$
F_{\epsilon}=\frac{1.75}{\sqrt{150 \epsilon^{3}}}, \quad K=\frac{\epsilon^{3} d_{m}^{2}}{150(1-\epsilon)^{2}},
$$

with $d_{m}$ being the ice droplet diameter.

During frosting, the evolution of the frost layer can be tracked by the ice volume fraction $f_{s}$, which is updated based on the mass conservation equation for the ice phase,

$$
\rho_{s} \frac{\partial f_{s}}{\partial t}=\dot{m},
$$

with $\rho_{s}$ being the ice density. It is obvious that $\dot{m}$ is the key factor in the frosting simulations, and the calculation of $\dot{m}$ is described by [20],

$$
\dot{m}=\tau_{v i} \in \rho_{l} W_{v} \frac{W_{v}-W_{v, \text { sat }}}{W_{v, \text { sat }}},
$$

where $\tau_{v i}$ is the time relaxation coefficient per second. As provided in the American Society of Heating, Refrigerating and Air-Conditioning Engineers (ASHRAE) handbook, the saturated humidity $W_{v \text {, sat }}$ and the water vapor saturation pressure $p_{\text {sat }}$ corresponding to $T$ can be calculated by empirical correlations as [39]

$$
W_{v, \text { sat }}(T)=0.622 \frac{p_{\text {sat }}(T)}{p_{0}-0.378 p_{\text {sat }}(T)}
$$

and

$$
p_{\text {sat }}(T)=\left\{\begin{array}{l}
\exp \left(a_{0} T^{-1}+a_{1}+a_{2} T+a_{3} T^{2}+a_{4} T^{3}+a_{5} T^{4}+a_{6} \ln T\right), \\
\text { for } T \in[173.15 \mathrm{~K}, 273.15 \mathrm{~K}], \\
\exp \left(b_{0} T^{-1}+b_{1}+b_{2} T+b_{3} T^{2}+b_{4} T^{3}+b_{5} \ln T\right), \\
\quad \text { for } T \in[273.15 \mathrm{~K}, 473.15 \mathrm{~K}],
\end{array}\right.
$$

respectively. Here $p_{0}$ is the atmospheric pressure, and the values of parameters $a$ and $b$ are given as

$$
\begin{aligned}
& a_{0}=-5.6745359 \times 10^{3}, \quad a_{1}=6.3925247, \quad a_{2}=-9.677843 \times 10^{-3}, \\
& a_{3}=6.2215701 \times 10^{-7}, \quad a_{4}=2.0747825 \times 10^{-9}, \\
& a_{5}=-9.484024 \times 10^{-13}, \quad a_{6}=4.1635019, \\
& b_{0}=-5.8002206 \times 10^{-3}, \quad b_{1}=1.3914993, \quad b_{2}=-4.8640239 \times 10^{-2}, \\
& b_{3}=4.1764768 \times 10^{-5}, \quad b_{4}=-1.4452093 \times 10^{-8}, \quad b_{5}=6.5459673 .
\end{aligned}
$$

Note that, in Eq. (14), the unit of $p_{\text {sat }}$ is pascal $(\mathrm{Pa})$ and $T$ is based on the Kelvin scale $(\mathrm{K})$, which can be converted from Celsius scale $\left({ }^{\circ} \mathrm{C}\right)$ by $T(\mathrm{~K})=T\left({ }^{\circ} \mathrm{C}\right)+273.15$.

It has been reported that, during the frosting process, the mass transferred from humid air can be divided into two parts. One for the frost layer growth at the frost surface and the other for frost densification within the frost layer [7]. To realize these actual frosting characteristics, a criterion for $\dot{m}$ is given as [20]

$$
\dot{m}= \begin{cases}\dot{m}, & \text { for } \quad \frac{W_{v}-W_{v, \text { sat }}}{W_{v, \text { sat }}} \geqslant B \frac{u}{u_{0}} \\ 0, & \text { for } \quad \frac{W_{v}-W_{v, \text { sat }}}{W_{v, \text { sat }}}<B \frac{u}{u_{0}}\end{cases}
$$

where $u=\left(u_{x}^{2}+u_{y}^{2}\right)^{1 / 2}$ is the scalar absolute value of velocity $\boldsymbol{u}$, with $u_{x}$ and $u_{y}$ being the $x$ and $y$ components of $\boldsymbol{u}$. The empirical correlation coefficient $B$ is calculated by [20]

$$
\begin{aligned}
B= & \left(-4.8 T_{w}^{2}+2489 T_{w}-3.21 \times 10^{5}\right) \\
& \times\left(0.687 u_{0}+1.771\right) W_{v, 0} .
\end{aligned}
$$

It is obvious that, within the frost layer or near the frost surface, the fluid velocity is small enough to satisfy the criterion $\frac{W_{v}-W_{v, \text { sat }}}{W_{v \text {,sat }}} \geqslant B \frac{u}{u_{0}}$, so that the mass transfer takes place. In the humid air region, however, the velocity is usually much larger and no frosting occurs. Note that the calculation for mass transfer rate in the present model can be conveniently replaced by other expressions if necessary.

In addition to the porosity $\epsilon$, the capacity ratio $\sigma$, and the mass fraction $W_{v}$, the above governing equations (3)-(6) are also characterized by the following nondimensional parameters:

$$
\begin{aligned}
\boldsymbol{u}^{*} & =\frac{\boldsymbol{u}}{U}, \quad T^{*}=\frac{T-T_{w}}{\Delta T}, \quad p^{*}=\frac{p}{\rho_{l} U^{2}}, \quad t^{*}=\frac{t}{L / U}, \\
\dot{m}^{*} & =\frac{\dot{m}}{\rho_{l} U / L}, \quad \boldsymbol{F}^{*}=\frac{\boldsymbol{F}}{U^{2} / L}, \quad \boldsymbol{M}^{*}=\frac{\boldsymbol{M}}{\rho_{l} U^{2} / L}, \quad \mathrm{Ju}=\frac{\nu_{e}}{v_{l}}, \\
\mathrm{Jt} & =\frac{\alpha_{e}}{\alpha_{l}}, \quad \mathrm{Jw}=\frac{D_{e}}{D_{l}}, \quad \mathrm{Re}=\frac{L U}{v_{l}}, \quad \mathrm{Pe}=\frac{L U}{D_{l}}, \\
\mathrm{Pr} & =\frac{\nu_{l}}{\alpha_{l}}, \quad \text { Ste }=\frac{c_{p, l} \Delta T}{L_{a}}, \quad \mathrm{Da}=\frac{K}{L^{2}},
\end{aligned}
$$

where the asterisked variables are the corresponding dimensionless ones, $\alpha_{l}$ and $D_{l}$ are the thermal diffusivity and the 
diffusion coefficient of humid air, respectively. $L, U$, and $\Delta T$ are characteristic length, velocity, and temperature difference, respectively. Note that some characteristic numbers are involved in this problem: the Reynolds number Re, the Peclet number Pe, the Prandtl number Pr, the Stefan number Ste, the Darcy number $\mathrm{Da}$, and the viscosity, thermal diffusivity, and diffusion coefficient ratios Ju, Jt, and $\mathrm{Jw}$. In terms of these variables, the dimensional governing equations read

$$
\begin{gathered}
\frac{\partial \epsilon}{\partial t^{*}}+\nabla \cdot \boldsymbol{u}^{*}=-\dot{m}^{*} \\
\frac{\partial \boldsymbol{u}^{*}}{\partial t^{*}}+\boldsymbol{u}^{*} \cdot \nabla\left(\frac{\boldsymbol{u}^{*}}{\epsilon}\right)=-\nabla\left(\epsilon p^{*}\right)+\frac{\mathrm{Ju}}{\operatorname{Re}} \nabla^{2} \boldsymbol{u}^{*}+\boldsymbol{F}^{*}-\boldsymbol{M}^{*} \\
\sigma \frac{\partial T^{*}}{\partial t^{*}}+\boldsymbol{u}^{*} \cdot \nabla T^{*}=\frac{\mathrm{Jt}}{\operatorname{PrRe}} \nabla^{2} T-\frac{1}{\mathrm{Ste}} \frac{\partial \epsilon}{\partial t^{*}} \\
\epsilon \frac{\partial W_{v}}{\partial t^{*}}+\boldsymbol{u} \cdot \nabla W_{v}=\frac{\mathrm{Jw}}{\mathrm{Pe}} \nabla^{2} W_{v}-\dot{m}^{*}
\end{gathered}
$$

It can be seen that, as $\epsilon \rightarrow 1$ (without porous matrix), the present generalized equations (3)-(6) reduce to the NavierStokes equations and the heat and mass transfer equations for humid air. The frosting properties in the humid air region can be then described. Simultaneously, physical properties of the frost layer are defined as $0<\epsilon<1$ (with porous media). Thus, the frosting properties of both the humid air region and the frost layer can be described in a unified pattern. It also should be emphasized that, by setting the velocity field as $\boldsymbol{u}=(0,0)$, the convection effects can be neglected, and the present governing Eqs. (3)-(6) turn into those for the frost layer in the aforementioned second group of frosting models. In the meantime, the governing equations for the humid air region in the second group can be obtained from Eqs. (3)-(6) with $\epsilon \rightarrow 1$. So, by setting the porosity as $\epsilon \rightarrow 1$ and the velocity field as $\boldsymbol{u}=(0,0)$, respectively, the present Eqs. (3)-(6) will reduce to the governing equations in the second group of models. In addition, based on the volumeaveraging method, two sets of governing equations for the ice and air phases in the third group of models can be transferred into the present Eqs. (3)-(6) as conducted in Ref. [21]. In conclusion, the second and third groups of existing frosting models can be viewed as two specific cases of the present generalized one.

\section{GENERALIZED LB MODEL FOR FROSTING}

The LB method, originating from the lattice gas automata method, can be viewed as a special discrete scheme for the Boltzmann equation with discrete velocities [5]. In this method, the fluid properties are modeled by discrete Boltzmann equations describing the evolutions of particle distribution functions. Through the Chapman-Enskog procedure, the correct macroscopic equations of the fluid can be derived from the LB equations by defining the collision operators appropriately. On the basis of the LB model proposed by Guo et al. [31], a new generalized LB model is developed to solve the present governing equations (3)-(6) for the frosting process. This LB model is quite different from the existing ones for phase change processes in porous media from the following three aspects. First, due to the additional mass source $\dot{m}$ and momentum transfer rate $\boldsymbol{M}$, the LB equation for fluid flow needs to be modified. Second, an additional LB equation should be provided for the humidity field. Finally, $f_{s}$ is determined by the mass conservation equation (11), which is related to both temperature and humidity fields. The two-dimensional nine-velocity (D2Q9) LB model is applied, and the corresponding discrete velocities $\boldsymbol{e}_{i}$ and weight coefficients $w_{i}$ are [40]

$$
\begin{aligned}
\boldsymbol{e}_{i} & =e(0,0), \quad w_{i}=\frac{4}{9}, \quad i=0 ; \\
\boldsymbol{e}_{i} & =e\left[\cos \frac{(i-1) \pi}{2}, \sin \frac{(i-1) \pi}{2}\right], \quad w_{i}=\frac{1}{9}, \quad i=1-4 ; \\
\boldsymbol{e}_{i} & =\sqrt{2} e\left[\cos \frac{(2 i-1) \pi}{4}, \sin \frac{(2 i-1) \pi}{4}\right], \\
w_{i} & =\frac{1}{36}, \quad i=5-8 ;
\end{aligned}
$$

where $e=\delta_{x} / \delta_{t}$ is the lattice speed, with $\delta_{x}$ and $\delta_{t}$ denoting the lattice spacing and the time step, respectively. In this work, the parameter $e$ is given as the velocity unit, i.e., $e=1$.

\section{A. LB equation for the flow field}

Compared with the original LB model for the flow field [5], the additional mass source is considered here for frosting. This subsequently brings changes in both the evolution equation and the calculation of macroscopic quantities. The present LB equation for the flow field can be written as

$$
\begin{aligned}
f_{i}(\boldsymbol{x} & \left.+\boldsymbol{e}_{i} \delta_{t}, t+\delta_{t}\right)-f_{i}(\boldsymbol{x}, t) \\
= & -\frac{1}{\tau_{u}}\left[f_{i}(\boldsymbol{x}, t)-f_{i}^{\mathrm{eq}}(\boldsymbol{x}, t)\right]+\delta_{t} F_{i}+\delta_{t} R_{i},
\end{aligned}
$$

for $i=0,1, \ldots, 8$, where $f_{i}(\boldsymbol{x}, t)$ is the density distribution function for particles moving with discrete velocity $\boldsymbol{e}_{i}$ at position $\boldsymbol{x}$ and time $t$, and $f_{i}^{\mathrm{eq}}$ is the corresponding equilibrium distribution function,

$$
f_{i}^{\mathrm{eq}}=w_{i}\left\{\rho_{p}+\rho_{l}\left[\frac{\boldsymbol{e}_{i} \cdot \boldsymbol{u}}{c_{s}^{2}}+\frac{\boldsymbol{u} \boldsymbol{u}:\left(\boldsymbol{e}_{i} \boldsymbol{e}_{i}-c_{s}^{2} \boldsymbol{I}\right)}{2 \epsilon c_{s}^{4}}\right]\right\} .
$$

Here $\rho_{p}$ is a variable related to fluid pressure as $\rho_{p}=\epsilon p / c_{s}^{2}$, with $c_{s}=e / \sqrt{3}$ being the lattice sound velocity. This LB model for incompressible fluid flows can reduce compressible errors [40,41]. Different from the original model [5], the distribution function $F_{i}$ accounts for both the body force $\boldsymbol{F}$ and the source term $\boldsymbol{M}$ as

$$
\begin{aligned}
F_{i}= & w_{i}\left(1-\frac{1}{2 \tau_{u}}\right) \\
& \times\left[\frac{\boldsymbol{e}_{i} \cdot\left(\rho_{l} \boldsymbol{F}+\boldsymbol{M}\right)}{c_{s}^{2}}+\frac{\boldsymbol{u}\left(\rho_{l} \boldsymbol{F}+\boldsymbol{M}\right):\left(\boldsymbol{e}_{i} \boldsymbol{e}_{i}-c_{s}^{2} \boldsymbol{I}\right)}{\epsilon c_{s}^{4}}\right] .
\end{aligned}
$$

The newly introduced distribution function $R_{i}$ that stands for the mass source $\dot{m}$ is defined as

$$
R_{i}=-w_{i} \dot{m}\left(1-\frac{1}{2 \tau_{u}}\right) .
$$


At each time step, after the above evolution, the fluid density and velocity are obtained from the distribution functions as

$$
\rho=\sum_{i} f_{i}-\frac{\delta_{t}}{2} \dot{m}, \quad \rho_{l} \boldsymbol{u}=\sum_{i} \boldsymbol{e}_{i} f_{i}+\frac{\delta_{t}}{2}\left(\rho_{l} \boldsymbol{F}+\boldsymbol{M}\right) .
$$

It is noted that the total body force $\boldsymbol{F}$ also contains the velocity $\boldsymbol{u}$, and thus the calculation of $\boldsymbol{u}$ in Eq. (28) is nonlinear and can be rewritten as

$$
\rho_{l} \boldsymbol{u}=\frac{\boldsymbol{v}}{c_{0}+\sqrt{c_{0}^{2}+c_{1}|\boldsymbol{v}|}},
$$

with

$$
\begin{aligned}
\boldsymbol{v} & =\sum_{i} \boldsymbol{e}_{i} f_{i}+\rho_{l} \frac{\delta_{t}}{2} \epsilon \boldsymbol{G}+\frac{\delta_{t}}{2} \boldsymbol{M}, \\
c_{0} & =\frac{1}{2}\left(1+\epsilon \frac{\delta_{t}}{2} \frac{v_{l}}{K}\right), \quad c_{1}=\epsilon \frac{\delta_{t}}{2} \frac{F_{\epsilon}}{\sqrt{K}} .
\end{aligned}
$$

The governing equations (3) and (4) for the flow field can be recovered exactly through the Chapman-Enskog analysis on the LB equation (24). To achieve this, the relaxation time $\tau_{u}$ must be related to the effective kinematic viscosity $v_{e}$ by

$$
v_{e}=c_{s}^{2}\left(\tau_{u}-\frac{1}{2}\right) \delta_{t}
$$

The details of this derivation are given in Appendix A.

\section{B. LB equation for the temperature field}

For the temperature field, another LB equation is developed to determine the temperature distribution function $g_{i}(\boldsymbol{x}, t)$,

$$
\begin{aligned}
& g_{i}\left(\boldsymbol{x}+\boldsymbol{e}_{i} \delta_{t}, t+\delta_{t}\right)-g_{i}(\boldsymbol{x}, t) \\
& \quad=-\frac{1}{\tau_{t}}\left[g_{i}(\boldsymbol{x}, t)-g_{i}^{\mathrm{eq}}(\boldsymbol{x}, t)\right]+\delta_{t} G_{i},
\end{aligned}
$$

where $\tau_{t}$ is the relaxation time and $g_{i}^{\mathrm{eq}}$ is the equilibrium distribution function,

$$
g_{i}^{\mathrm{eq}}=w_{i} T\left(\sigma+\frac{\boldsymbol{e}_{i} \cdot \boldsymbol{u}}{c_{s}^{2}}\right) .
$$

The source term $G_{i}$ in Eq. (32) is taken as

$$
G_{i}=-w_{i} \sigma \frac{L_{a}}{c_{p, l}} \frac{\partial \epsilon}{\partial t},
$$

of which the time derivative term is treated by the backwarddifference scheme [42],

$$
\frac{\partial \epsilon}{\partial t}=\frac{\epsilon(\boldsymbol{x}, t)-\epsilon\left(\boldsymbol{x}, t-\delta_{t}\right)}{\delta_{t}}
$$

In this model, the temperature is defined as

$$
\sigma T=\sum_{i} g_{i}
$$

Through the Chapman-Enskog analysis, the governing equation (5) for the temperature field can also be recovered, with the effective thermal diffusion coefficient being

$$
\alpha_{e}=\sigma c_{s}^{2}\left(\tau_{t}-\frac{1}{2}\right) \delta_{t}
$$

\section{LB equation for the humidity field}

The LB model for the humidity field is similar to that for the temperature field, since their governing equations are of the same pattern. The evolution equation reads

$$
\begin{aligned}
h_{i} & \left(\boldsymbol{x}+\boldsymbol{e}_{i} \delta_{t}, t+\delta_{t}\right)-h_{i}(\boldsymbol{x}, t) \\
\quad= & -\frac{1}{\tau_{w}}\left[h_{i}(\boldsymbol{x}, t)-h_{i}^{\mathrm{eq}}(\boldsymbol{x}, t)\right]+\delta_{t} H_{i},
\end{aligned}
$$

where $h_{i}(\boldsymbol{x}, t)$ denotes the humidity distribution function, $\tau_{w}$ is the relaxation time, and $h_{i}^{\mathrm{eq}}$ is the equilibrium distribution function,

$$
h_{i}^{\mathrm{eq}}=w_{i} W_{v}\left(\epsilon+\frac{\boldsymbol{e}_{i} \cdot \boldsymbol{u}}{c_{s}^{2}}\right) .
$$

The source term $H_{i}$ in the evolution equation (38) is given by

$$
H_{i}=-w_{i} \epsilon \frac{\dot{m}}{\rho_{s}} .
$$

Again, the governing equation (6) can be recovered from the LB equation (38), using the Chapman-Enskog procedure. The effective humidity diffusion coefficient is defined as

$$
D_{e}=\epsilon c_{s}^{2}\left(\tau_{w}-\frac{1}{2}\right) \delta_{t},
$$

and the mass fraction of water vapor is calculated by

$$
\epsilon W_{v}=\sum_{i} h_{i} .
$$

The derivation details of the Chapman-Enskog analysis on LB equations for both temperature and humidity fields can be found in Appendix B.

\section{VALIDATION}

In this section, numerical simulations are carried out to demonstrate the capability and reliability of the present generalized LB model. The problems considered here include the conduction solidification in a semi-infinite space, the melting with natural convection in a porous medium, and the frosting of humid air in a parallel channel. The present results are compared with corresponding numerical, analytical, and experimental data in previous studies.

\section{A. Conduction solidification in a semi-infinite space}

The present LB model is first validated by simulating the conduction-dominated solidification in a semi-infinite space, which can be viewed as a classical Stefan problem for solidification [43]. As displayed in Fig. 1, the computational domain is $0 \leqslant x \leqslant l_{x}$ and $0 \leqslant y \leqslant l_{y}$, and it is initially filled

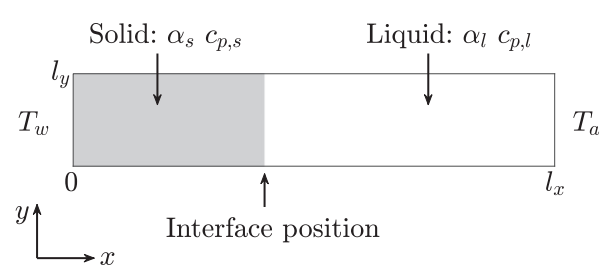

FIG. 1. The computational domain of the conduction solidification problem. 
with liquid material at temperature $T_{a}\left(T_{a}>T_{m}\right.$, where $T_{m}$ is the solidification temperature). Then, the left wall temperature is lowered to $T_{w}\left(T_{w}<T_{m}\right)$. This subsequently introduces the solidification of liquid material at the surface $x=0$, and the solid-liquid interface moves toward the liquid phase. The boundary conditions are given as follows:

$$
\begin{aligned}
& T(0, y, t)=T_{w}, \quad T\left(l_{x}, y, t\right)=T_{a}, \\
& T(x, 0, t)=T\left(x, l_{y}, t\right) .
\end{aligned}
$$

The fixed temperature walls are realized by the nonequilibrium extrapolation scheme [40], and the periodic condition is imposed at the top and bottom boundaries. The convection is not considered, so the velocity field is set to be zero consistently. For this problem, the temperature distribution can be obtained analytically [43],

$T(\boldsymbol{x}, t)= \begin{cases}T_{w}+\frac{\left(T_{m}-T_{w}\right) \operatorname{erf}\left(x / 2 \sqrt{\alpha_{s} t}\right)}{\operatorname{erf}(\eta)}, & 0<x \leqslant x_{m}(t), \\ T_{a}+\frac{\left(T_{m}-T_{w}\right) \operatorname{erfc}\left(x / 2 \sqrt{\alpha_{l} t}\right)}{\operatorname{erfc}\left(\eta \sqrt{\alpha_{s} / \alpha_{l}}\right)}, & x_{m}(t)<x \leqslant l_{x},\end{cases}$

where $\operatorname{erf}(z)=(2 / \sqrt{\pi}) \int_{0}^{z} \exp \left(-\theta^{2}\right) d \theta$ is the error function, $\operatorname{erfc}(z)=1-\operatorname{erf}(z)$ is the complementary error function, and $x_{m}(t)$ is the averaged position of the solid-liquid interface. The parameter $\eta$ is determined by [43]

$$
\begin{aligned}
& \frac{\exp \left(-\eta^{2}\right)}{\operatorname{erf}(\eta)}+\frac{\lambda_{l}}{\lambda_{s}}\left(\frac{\alpha_{s}}{\alpha_{l}}\right)^{1 / 2} \frac{T_{m}-T_{a}}{T_{m}-T_{w}} \frac{\exp \left(-\eta^{2} \alpha_{s} / \alpha_{l}\right)}{\operatorname{erfc}\left(\eta \sqrt{\alpha_{s} / \alpha_{l}}\right)} \\
& =\frac{\eta L_{a} \sqrt{\pi}}{c_{p, s}\left(T_{m}-T_{w}\right)} .
\end{aligned}
$$

In this simulation, the domain size and lattice size are $l_{x} \times$ $l_{y}=800 \times 10$ and $N_{x} \times N_{y}=800 \times 10$, respectively. The required parameters are set as $T_{a}=2, T_{m}=1, T_{w}=0, \alpha_{s}=$ $0.005, \sigma=1, c_{p, l}=c_{p, s}=1, \rho_{l}=\rho_{s}=1$, Ste $=2, \boldsymbol{G}=$ $(0,0), \alpha_{e}=f_{s} \alpha_{s}+\epsilon \alpha_{l}$. The parameter $f_{s}$ is updated as that in Ref. [35]. It is noted that the physical parameters are all expressed in lattice units, and the characteristic length, velocity, and temperature difference are chosen as

$$
L=l_{x} / 8, \quad U=\alpha_{s} / L, \quad \Delta T=T_{a}-T_{m} .
$$

The temperature profiles for different values of thermal diffusivity ratio $\alpha_{l} / \alpha_{s}$ are obtained by the present model, and the results at $t^{*}=1$ are plotted in Fig. 2. It can be observed that the present numerical results are in good agreement with the analytical solutions. Besides, the global relative error between numerical and analytical results is calculated as

$$
E=\frac{\sum\left|T_{\text {num }}-T_{\text {ana }}\right|}{\sum T_{\text {ana }}},
$$

with $T_{\text {num }}$ and $T_{\text {ana }}$ being the the numerical and the analytical solutions, respectively. The calculated errors for $\alpha_{l} / \alpha_{s}=$ $1,2,10$ are $0.61 \%, 0.35 \%$, and $1.07 \%$, respectively, and this quantitative comparison shows a satisfactory agreement again.

\section{B. Convection melting in a porous medium}

The effects of convection are not involved in the above test, thus the model is then validated by simulating melting coupled

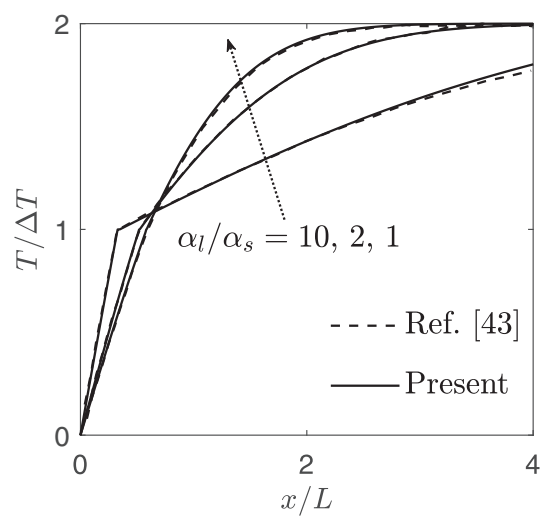

FIG. 2. Temperature profiles comparison against analytical solutions in Ref. [43].

with convection in a porous medium with an initial porosity $\epsilon_{0}$ and density $\rho_{d}$ (see Fig. 3). Initially, the solid material occupies the region with temperature $T_{w}\left(T_{w}<T_{m}\right)$, and then the temperature of the left wall is raised to $T_{a}\left(T_{a}>T_{w}\right)$. Consequently, melting begins along the left wall and proceeds inside the porous medium. The boundary conditions are

$$
\begin{array}{rlrl}
\boldsymbol{u}(0, y, t) & =(0,0), & T(0, y, t) & =T_{a}, \\
\boldsymbol{u}\left(l_{x}, y, t\right) & =(0,0), & T\left(l_{x}, y, t\right)=T_{w}, \\
\frac{\partial \boldsymbol{u}}{\partial y}(x, 0, t) & =(0,0), & \frac{\partial T}{\partial y}(x, 0, t)=0, \\
\frac{\partial \boldsymbol{u}}{\partial y}\left(x, l_{y}, t\right) & =(0,0), & \frac{\partial T}{\partial y}\left(x, l_{y}, t\right)=0 .
\end{array}
$$

The top and bottom adiabatic walls are realized by the halfway bounce-back scheme [40], while the inlet and outlet are treated with the nonequilibrium extrapolation scheme.

The simulation parameters are set to be the same as used in Ref. [44], namely $l_{x} \times l_{y}=200 \times 200, N_{x} \times N_{y}=$ $200 \times 200, \quad \epsilon_{0}=0.385, \quad \mathrm{Da}=1.37 \times 10^{-5}, \quad \mathrm{Ju}=1, \quad \mathrm{Pr}=$ $0.0208, \mathrm{Jt}=0.2719$, Ste $=0.1241, \alpha_{l}=\alpha_{s}=0.005, \rho_{l}=$ $\rho_{s}=\rho_{d}=1, T_{a}=45, T_{m}=29.78, T_{w}=20, \sigma_{l}=0.8604$, $\sigma_{s}=0.8352$. Based on the Boussinesq approximation, the buoyancy force is introduced by $\boldsymbol{G}=g \beta\left(T-T_{w}\right) \boldsymbol{j}$, where $g$ is the gravitational acceleration, and the related Rayleigh

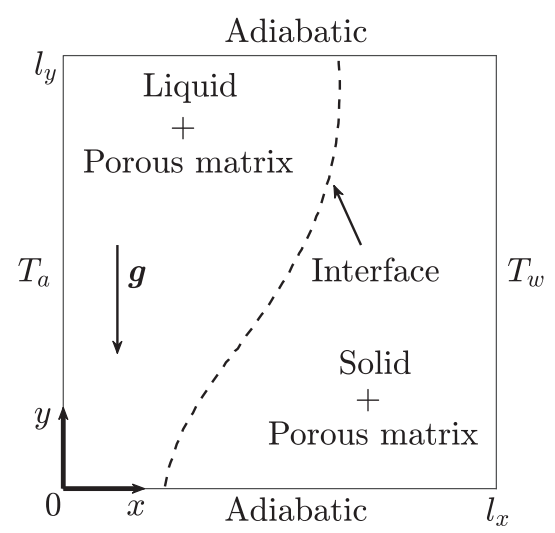

FIG. 3. The computational domain of the convection melting problem. 


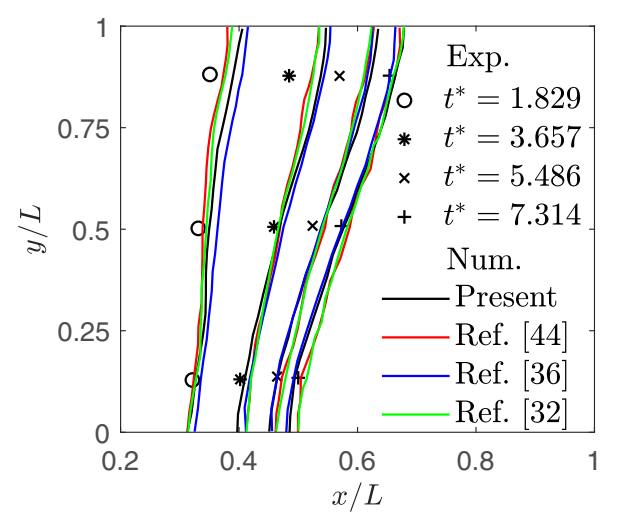

FIG. 4. Comparison of numerical interface locations with experimental data in Ref. [44].

number is $\mathrm{Ra}=g \beta \Delta T L^{3} / \nu_{l} \alpha_{l}=8.409 \times 10^{5}$. These parameters are also given in lattice units, and $f_{s}$ is calculated as that in the above case. The characteristic parameters are chosen as

$$
L=l_{y}, \quad U=\sqrt{g \beta \Delta T L}, \quad \Delta T=T_{a}-T_{w} .
$$

Figure 4 provides the melting interface profiles at different time instants. The present numerical results are compared with the experimental and numerical data from Beckermann and Viskanta [44], and with numerical results predicted by two existing LB models in Refs. [32] and [36] as well. It is observed that both the present and previous numerical results match reasonably well with the experimental data, and the calculated global relative errors between experimental and numerical results from the present LB model and those in Refs. [44], [32], and [36] are 3.95\%, 3.27\%, 3.66\%, and $4.75 \%$, respectively. However, some discrepancies can be observed at early time instants, which can be explained by the uncertainties in the measurements and effective parameters as pointed out by Beckermann and Viskanta [44].

\section{Frosting of humid air in a parallel channel}

The proposed LB model is finally validated by calculating the frost growth on cold plates. The computational domain is $0 \leqslant x \leqslant l_{x}$ and $0 \leqslant y \leqslant l_{y}$, which is structured by two parallel impermeable stationary walls (see Fig. 5). The top plate and the leading part of bottom plate $\left(0 \leqslant x<l_{x 1}\right)$ are adiabatic, while the rear part of bottom wall $\left(l_{x 1} \leqslant x \leqslant l_{x}\right)$ is fixed at temperature $T_{w}$, which is lower than the dew point. The humid air flows through the channel from the inlet, and the frost layer appears and grows on the cold wall. The boundary

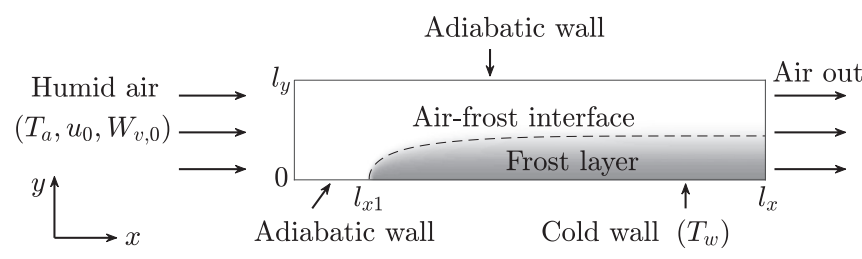

FIG. 5. The computational domain of the frosting problem.
TABLE I. Simulation conditions for the frosting validation problem.

\begin{tabular}{lccc}
\hline \hline No. & \multicolumn{2}{c}{ Parameters } & Results \\
\hline \multirow{4}{*}{$T_{w}=-19.5^{\circ} \mathrm{C}$} & $T_{a}=21.4^{\circ} \mathrm{C}$ & \\
Test 1 & $W_{v, 0}=0.0062 \mathrm{~kg} / \mathrm{kg}$ & $u_{0}=0.6 \mathrm{~m} / \mathrm{s}$ & Fig. 6 \\
& $l_{x}=140 \mathrm{~mm}$ & $l_{y}=10 \mathrm{~mm}$ & \\
& $l_{x 1}=20 \mathrm{~mm}$ & $N_{x} \times N_{y}=1400 \times 100$ & \\
& $T_{w}=-13^{\circ} \mathrm{C}$ & $T_{a}=2^{\circ} \mathrm{C}$ & \\
Test 2 & $W_{v, 0}=0.0037 \mathrm{~kg} / \mathrm{kg}$ & $u_{0}=0.92 \mathrm{~m} / \mathrm{s}$ & Fig. 7 \\
& $l_{x}=75 \mathrm{~mm}$ & $l_{y}=5 \mathrm{~mm}$ & \\
& $l_{x 1}=25 \mathrm{~mm}$ & $N_{x} \times N_{y}=750 \times 50$ & \\
\hline \hline
\end{tabular}

conditions are

$$
\begin{aligned}
T(0, y, t) & =T_{a}, \quad \boldsymbol{u}(0, y, t)=\left(u_{0}, 0\right), \\
W_{v}(0, y, t) & =W_{v, 0}, \quad \frac{\partial T}{\partial x}\left(l_{x}, y, t\right)=0, \\
\frac{\partial \boldsymbol{u}}{\partial x}\left(l_{x}, y, t\right) & =(0,0), \quad \frac{\partial W_{v}}{\partial x}\left(l_{x}, y, t\right)=0, \\
\frac{\partial T}{\partial y}\left(x, l_{y}, t\right) & =0, \quad \boldsymbol{u}\left(x, l_{y}, t\right)=(0,0), \\
\frac{\partial W_{v}}{\partial y}\left(x, l_{y}, t\right) & =0, \quad \boldsymbol{u}(x, 0, t)=(0,0), \\
\frac{\partial W_{v}}{\partial y}(x, 0, t) & =0, \quad \frac{\partial T}{\partial y}(x, 0, t)=0, x \in\left[0, l_{x 1}\right), \\
T(x, 0, t) & =T_{w}, x \in\left[l_{x 1}, l_{x}\right] .
\end{aligned}
$$

The adiabatic walls are implemented with the halfway bounce-back scheme, and the nonequilibrium extrapolation scheme is applied to the inlet and outlet and the cold wall as well. Here the characteristic length, velocity, and temperature difference are chosen as

$$
L=l_{y}, \quad U=u_{0}, \quad \Delta T=T_{a}-T_{w} .
$$

In order to compare with previous experimental data, the geometry as well as operating parameters are set as in Table I. Tests 1 and 2 follow the experiments in Refs. [14] and [20], respectively. The effective diffusion coefficient $D_{e}$ of the frost layer is determined by the empirical correlation [10],

$$
D_{e}=D_{l} \epsilon \frac{1+\epsilon}{2} .
$$

As for the calculation of effective thermal conductivity $\lambda_{e}$, a well-known correlation proposed by Yonko et al. [45] is employed,

$$
\lambda_{e}=0.024248+7.2311 \times 10^{-4} \rho_{f}+1.183 \times 10^{-6} \rho_{f}^{2},
$$

where the frost density $\rho_{f}$ is obtained using the volumeaveraging method in Eq. (2). This expression is based on experimental data and has been reported to be close to reality [17]. It should be noted that the mass transfer rate $\dot{m}$ is usually very small, and it appears at places with relatively small velocity. Thus, the momentum transfer caused by frost deposition can be neglected, namely $\boldsymbol{M} \approx(0,0)$ [21]. This assumption is also applied for the following frosting simulations. 


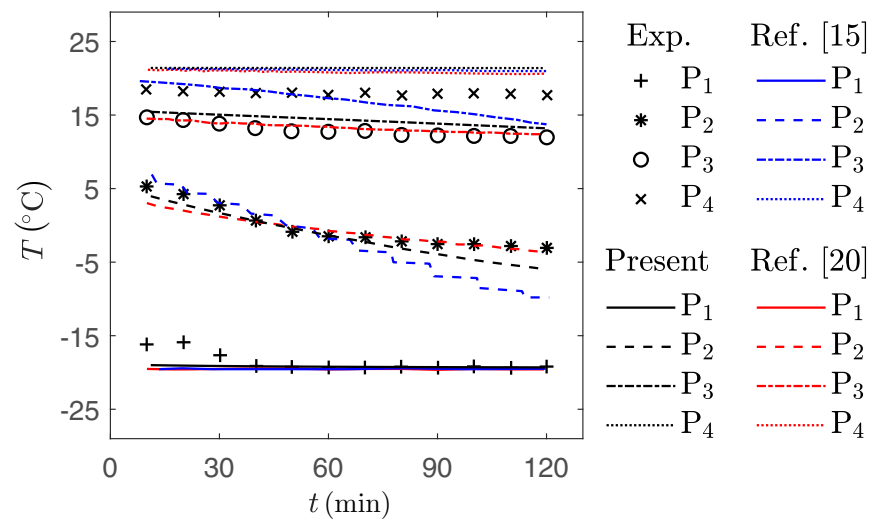

FIG. 6. Comparison of numerical temperature evolutions with experimental data in Ref. [14].

Test 1 in Table $\mathrm{I}$ is first simulated, and the temperature evolutions are measured against time at four specific points $\mathrm{P}_{1-4}$, with $x=130 \mathrm{~mm}$ and $y=0,3,6,9 \mathrm{~mm}$. In Fig. 6, the temperature evolutions simulated by the present LB model are represented with black lines, and the experimental data recorded by Lenic et al. [14] are plotted with symbols for comparison. In addition, to comprehensively validate the reliability of the present model, numerical results obtained by existing models of Armengol et al. [15] and $\mathrm{Wu}$ et al. [20], respectively, are shown in Fig. 6 as well. It is found that numerical results predicted by the three models show a fair agreement with the experimental data, and the calculated global relative errors between numerical and experimental results are provided in Table II. Note that the relative error of temperature is calculated based on the Kelvin scale. At points $\mathrm{P}_{1}$ and $\mathrm{P}_{4}$, the three models show a similar capability and accuracy to describe the frosting process, and the calculated relative errors are within the range of $0.2-1.2 \%$. Regarding the temperature evolutions at positions $\mathrm{P}_{2}$ and $\mathrm{P}_{3}$, excellent agreement is reached between experimental data and numerical results obtained by the present LB model and that in Ref. [20]. The calculated relative errors for these two models are all less than $0.6 \%$. Based on the model in Ref. [15], however, numerical results differ significantly from the experimental data, and the calculated errors are $0.99 \%$ and $1.44 \%$ for $\mathrm{P}_{2}$ and $\mathrm{P}_{3}$, respectively.

TABLE II. Comparison of global relative errors against the experimental data for the frosting validation problem.

\begin{tabular}{lccccc}
\hline \hline & & \multicolumn{3}{c}{ Models } & \\
\cline { 3 - 5 } & Points & Present & Ref. [20] & Ref. [15] & Results \\
\hline Test 1 & P1 & $0.27 \%$ & $0.4 \%$ & $0.35 \%$ & \\
& P2 & $0.44 \%$ & $0.31 \%$ & $0.96 \%$ & Fig. 6 \\
& P3 & $0.52 \%$ & $0.17 \%$ & $1.44 \%$ & \\
& P4 & $1.15 \%$ & $1.09 \%$ & $1.13 \%$ & \\
& \multicolumn{4}{c}{ Models } & \\
& Times & Present & Ref. [20] & Results \\
\hline Test 2 & 20 min & $9.47 \%$ & $12.97 \%$ & \multirow{2}{*}{ Fig. 7 } \\
& 40 min & $6.87 \%$ & $6.92 \%$ & \\
& 60 min & $11.52 \%$ & $7.23 \%$ & \\
\hline \hline
\end{tabular}

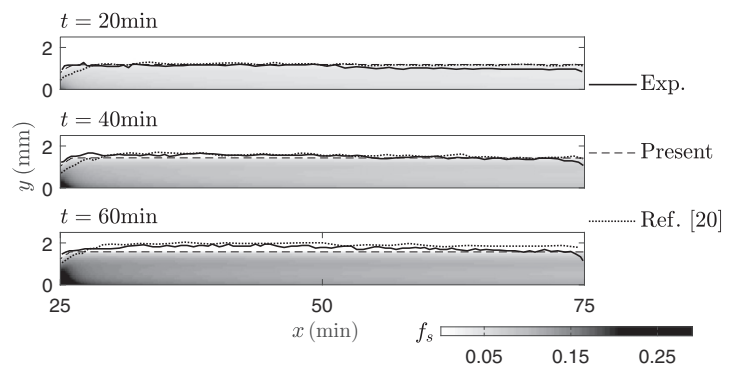

FIG. 7. Comparison of predicted frost layer height distributions with experimental data in Ref. [20].

Even though the evolution of local temperature has been predicted successfully by the present LB model, it is still necessary to verify the simulated frost thickness. Therefore, Test 2 in Table I is conducted simultaneously. Figure 7 presents the calculated frost layer distributions at different frosting time instants, and the frost surface at each time is marked by a black dash line. The experimental and numerical results reported by $\mathrm{Wu}$ et al. [20] are also provided for the sake of comparison. Again, the numerical results given by the present LB model and that in Ref. [20] are in good agreement with the experimental data, and the calculated relative errors are provided in Table II. It is found that the LB model can predict the frost layer much better than Ref. [20] at the first stage, while the model in Ref. [20] shows a slightly better performance at the later stage. It should be mentioned, however, that in the model of Ref. [20], physical properties of ice and humid air phases need to be calculated separately, and the realization of additional momentum and energy transfer conditions between two phases is required. In contrast, the present generalized LB model can automatically treat both phases in a unified formulation during the frosting process, and it turns out to be simpler and more efficient in predicting the frosting process correctly.

\section{FROSTING SIMULATIONS}

In this section, the frosting process of humid air in a parallel plate channel is studied. The computational domain is displayed in Fig. 5, and the geometry parameters are $l_{x}=$ $60 \mathrm{~mm}, l_{x 1}=10 \mathrm{~mm}, l_{y}=5 \mathrm{~mm}$. The boundary conditions are set as Eq. (50) and are realized as in the above frosting validation tests. This configuration permits horizontal frost growth, which is very common in industrial applications. Understanding of frosting on such a structure can also provide a basis for the frost formation over other complex surfaces. Specifically, the system is assumed to be neutrally buoyant and no external force is applied, i.e., $\boldsymbol{G}=(0,0)$. A set of numerical tests are considered as listed in Table III, and the characteristic length and velocity are chosen as Eq. (51).

Before proceeding further, the grid-independent test is first carried out by simulating the frosting process for Test A1, namely $T_{w}=-10^{\circ} \mathrm{C}, T_{a}=2^{\circ} \mathrm{C}, W_{v, 0}=0.004 \mathrm{~kg} / \mathrm{kg}, u_{0}=$ $1.0 \mathrm{~m} / \mathrm{s}$. The parallel channel in Fig. 5 is covered by three grids of sizes $N_{x} \times N_{y}=300 \times 25,600 \times 50$, and $1200 \times$ 100. The transverse distributions of $T$ at $x=25 \mathrm{~mm}$ and horizontal distributions of $f_{s}$ at $y=0.1 \mathrm{~mm}$ are recorded in 
TABLE III. Test conditions in the frosting simulations.

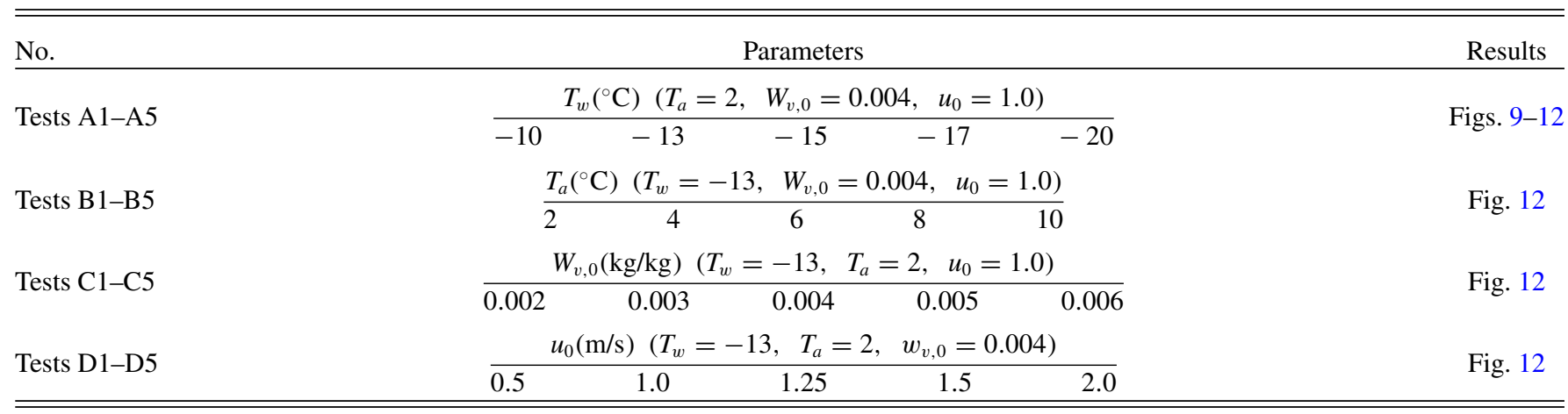

Fig. 8. It is observed that the results on the coarse grid $(300 \times$ 25) show some clear discrepancies from those on the other two finer grids. On the other hand, the $600 \times 50$ grid gives approximately the same results as the finest grid $1200 \times 100$. The above comparisons suggest that the $600 \times 50$ grid is fine enough to obtain grid-independent solutions, and it will be employed for the following simulations.

\section{A. Frosting phenomena}

The general phenomena of the frosting process are first investigated for the test conditions in Table III. It is found that the frosting behaviors under different conditions are in a similar fashion. As an example, Fig. 9 depicts the numerical results for Test A2, including the distributions of temperature, ice volume fraction, and velocity. These results can reflect the typical characteristics of the frosting process. It should be pointed out that the horizontal region in Fig. 9 is $5 \mathrm{~mm} \leqslant x \leqslant$ $60 \mathrm{~mm}$, and the length of the $y$ axis is enlarged 1.8 times to show the results clearly.

Figure 9(1) presents the temperature distributions for different time instants. The frost geometry at each time is also included with a black dash line. It is found that the cold region grows with time. This is because cooling of humid air is caused by the heat transferred from the cold wall and the frost layer that grows with time. To quantify the temperature field better, Fig. 10 plots the transverse temperature distribution varying with time at position $x=35 \mathrm{~mm}$. As reported by $\mathrm{Wu}$ et al. [20], the temperature decreases with time at a certain position due to the growth of the cold region [see Fig. 9(1)]. Besides, every temperature curve has an inflection point, which represents the air-frost interface. The temperature changes nearly linearly inside the frost layer, but nonlinearly in the air region. This can be explained by the fact that the heat is mainly transferred by conduction in the frost layer since the flow velocity is small [see Fig. 9(3)], while it is by convection in the humid air region. In addition, both the position and temperature of every inflection point increase with time. It is because the growth of the frost layer enhances the thermal resistance, which leads to increased frost surface temperature.

Figure 9(2) shows the evolution of $f_{s}$, which can reflect the development of the frost layer. It is shown that frost appears firstly at the leading part of the cold wall [see Fig. 9(2a)], then it grows continuously and finally distributes on the whole cold wall unevenly. Besides, the value of $f_{s}$ is obviously larger in the upstream part and the bottom region. These results match well with previous findings $[15,20]$. Note that, according to Eq. (2), the distribution of frost density $\rho_{f}$ will follow the same pattern as that of $f_{s}$. This means that the denser frost layer can be found in the leading edge and near the cold plate as well.

The geometries of the frost layer at various time instants are portrayed in Fig. 11(a). The outlines clearly show that the frost layer grows horizontally on the plate less than $l_{x 1}$. This entrance effect has been reported based on an additional 2D frost growth treatment in Ref. [15], but it can be realized automatically in the present model. Moreover, the frost thick-
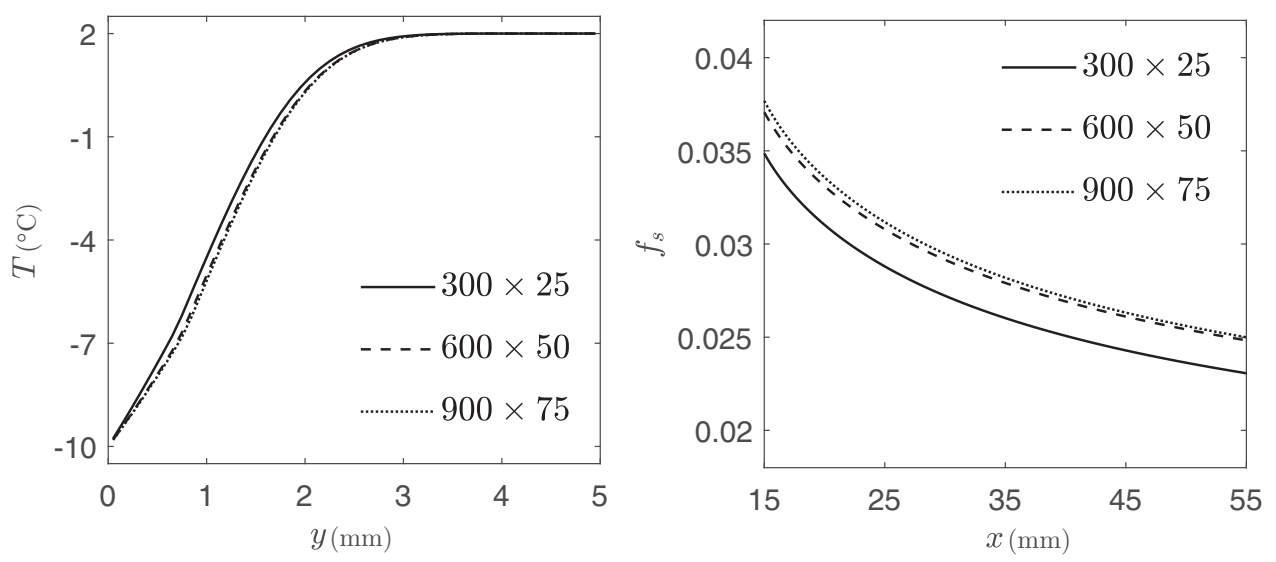

FIG. 8. Grid convergence of the spatial evolutions of $T$ and $f_{s}$ at $t=10 \mathrm{~min}$. 


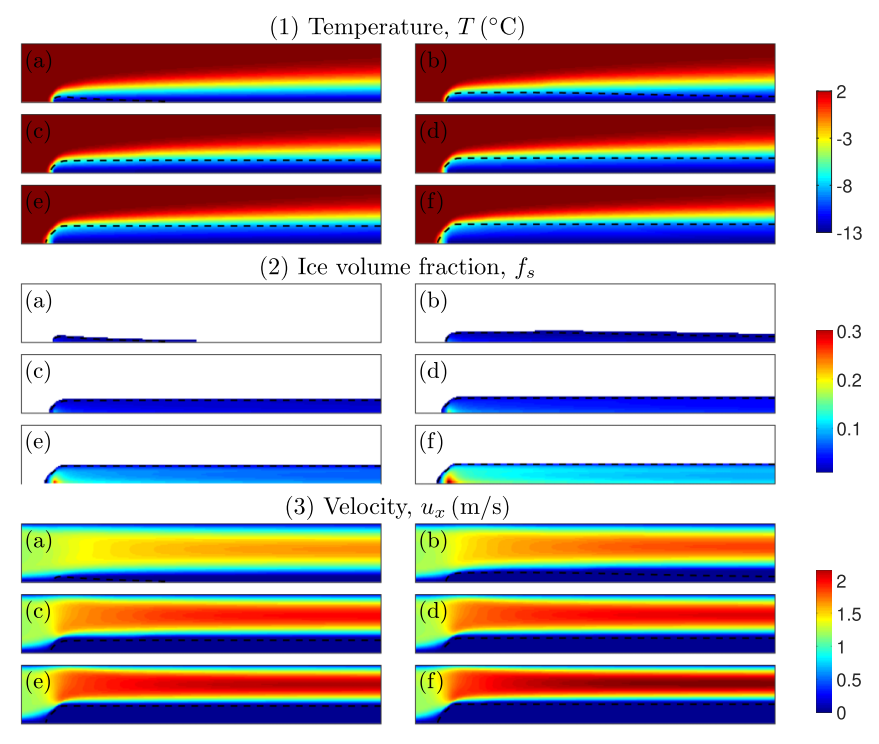

FIG. 9. Temperature, ice volume fraction, and velocity distributions for Test A2 at time instants $t=2.5,5,10,20,40,60 \mathrm{~min}$ from (a) to (f).

ness grows significantly in the first 20 mins, since the mass transferred from humid air is mainly used for frost growth in this period. However, the frost surface temperature increases with frost thickness [see Fig. 10(a)]. Thus when a certain value is reached, the main part of the transferred mass will be used for frost densification.

In addition, the horizontal distributions of $W_{v}$ and $f_{s}$ at $y=$ $0.1 \mathrm{~mm}$ for different frosting time instants are also recorded

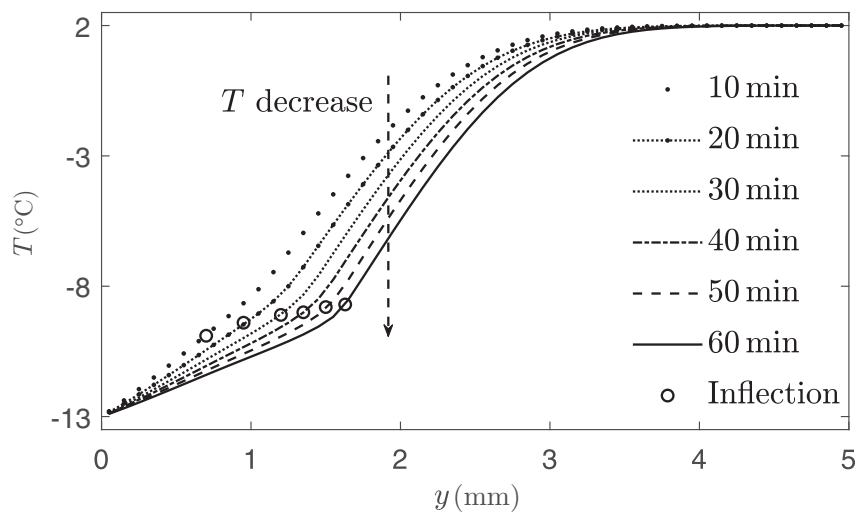

FIG. 10. Transverse temperature distributions at $x=35 \mathrm{~mm}$.

quantitatively in Figs. 11(b) and 11(c). The results for $W_{v}$ in Fig. 11(b) follow a similar pattern at different time instants. $W_{v}$ is higher at the frontal area and gets lower along the air flow direction. Based on Eq. (12), it can be concluded that a larger amount of mass will transfer to the frost layer at the leading edge. This subsequently introduces the denser frost layer in the frontal part but the looser frost layer in the rear part [see Fig. 9(2)]. The distributions of $f_{s}$ in Fig. 11(c) also show this trend, namely that every curve reaches its maximum value at the frontal position. Moreover, it is found that the difference between the values of $f_{s}$ at the leading and rear positions becomes larger. It is because more water vapor transfers into ice droplet at the frontal part, and this effect accumulates with time.
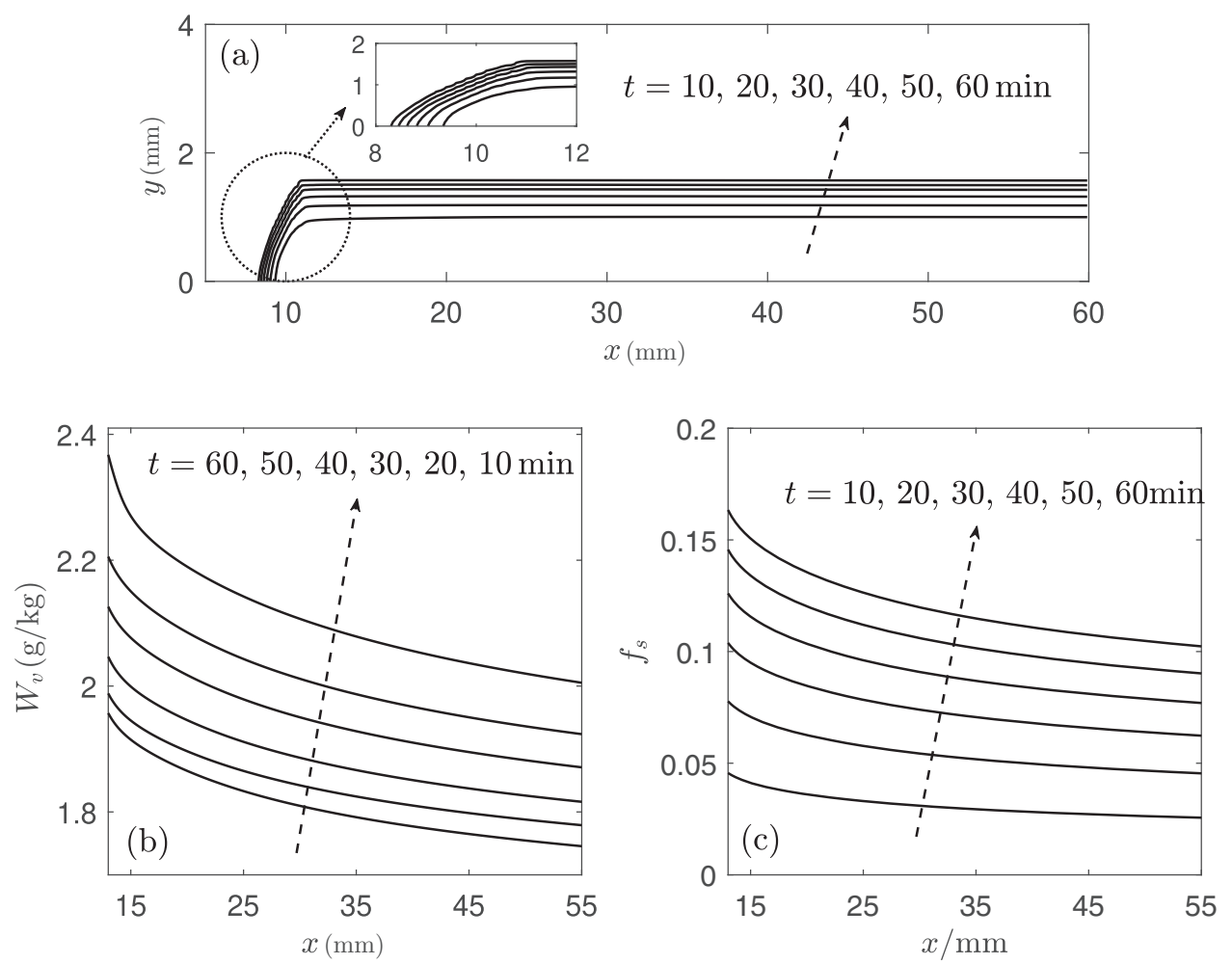

FIG. 11. Outlines of the frost layer and humidity and ice volume fraction distributions for various time at $y=0.1 \mathrm{~mm}$. 

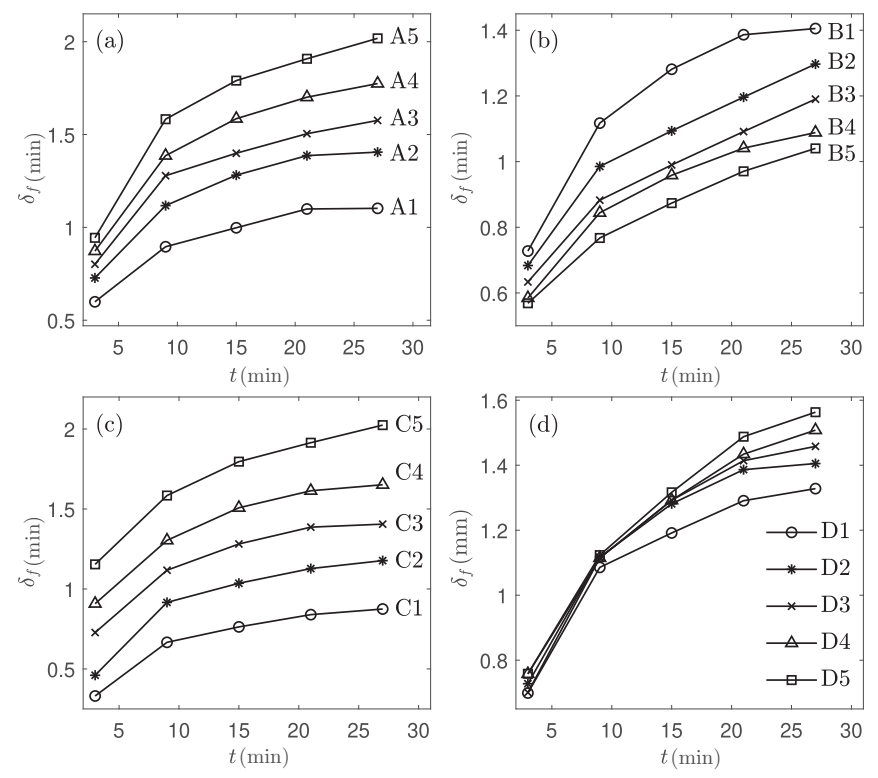

FIG. 12. Time evolutions of $\delta_{f}$ for Tests A-D in a parallel channel.

Finally, the velocity distributions are displayed in Fig. 9(3), and the frost geometry is also plotted by black dash line. It is observed that there is very little humid air flowing within the frost layer because of the matrix drag force. Besides, in the central flow region, the maximal velocity can be obtained, and this maximum value grows with time. This can be expected since the growing frost layer blocks the humid air flow passage.

\section{B. Influence factors}

After the observation of general phenomena, the analysis of the influence factors is also carried out. As mentioned in Refs. [46,47], the cold wall temperature $T_{w}$, the inlet air temperature $T_{a}$, humidity $W_{v, 0}$, and velocity $u_{0}$ are important parameters that affect frost layer growth on cold surface. Full understanding of their roles may contribute to developing defrosting techniques by changing the characteristics of the moist air and cold surfaces. Thus, as summarized in Table III, Tests A-D are performed to evaluate these influence factors. Here the average frost layer thickness $\delta_{f}$ is introduced as

$$
\delta_{f}=\frac{\sum_{N_{x 1}}^{N_{x}} \delta_{f, n}}{N_{x}-N_{x 1}}
$$

where $\delta_{f, n}$ stands for local thickness of the frost layer, and $N_{x 1}$ is the number of grids distributed on the bottom adiabatic wall. The parameter $\delta_{f}$ can be regarded as an authentic indicator for frost growth.

The time evolutions of $\delta_{f}$ for Tests A-D are presented in Fig. 12. Based on Eq. (12), it can be expected that larger mass fraction $W_{v}$ or smaller saturated mass fraction $W_{v \text {,sat }}$ will enhance the ice droplets deposition and subsequently will lead to faster growth of the frost layer. The numerical measurements shown in Figs. 12(a)-12(c) confirm this prediction. That is, the lower $T_{a}$ or $T_{w}$ and the higher $W_{v, 0}$ are favorable for the frost growth. It is because the lower $T_{a}$ or $T_{w}$ under constant $W_{v, 0}$ represents smaller saturated mass fraction $W_{v \text {,sat }}$, and the higher $W_{v, 0}$ introduces larger $W_{v}$. The time-evolution behaviors of $\delta_{f}$ for different values of $u_{0}$ are presented in Fig. 12(d). It is shown that the frost layer grows faster with larger inlet air velocity $u_{0}$ for the analyzed range. This is because the fast air velocity leads to a higher heat and mass transfer rate. It also should be emphasized, however, that the influence of $u_{0}$ is not so significant compared with other factors. Note that, in order to guarantee the appearance of frost layer, and to avoid the turbulence effects as well, much lower or higher values of air velocity $u_{0}$ are not further considered here.

To summarize, a series of tests have been conducted to study the frosting process in a parallel channel. Both the frosting characteristics and the influence factors can be correctly predicted. This suggests that the present LB model is suitable for simulating the frosting process on cold surfaces.

\section{CONCLUSIONS}

A generalized LB model has been proposed for the frosting process over cold surfaces. In this model, the velocity, temperature, and humidity fields are modeled by three separate LB equations. The frosting process is incorporated into the model by introducing the mass transfer term from humid air to ice phase. The attractive feature of this model is its capability to simulate the frosting properties in both the humid air and the frost layer simultaneously. Validation simulations have been carried out, including conduction solidification, convection melting, and the frosting process. It is observed that the numerical results match well with the analytical or experimental data, which demonstrates the reliability of the present LB model.

A series of numerical simulations of the frosting process in a parallel channel are carried out. Both spatial and temporal properties of the frost layer are obtained. The results have shown that the frost thickness increases with time, and the denser frost locates at the leading edge and near the cold surface as well. The air velocity within the frost layer is pretty small. However, it is much larger and increases with time in the humid air region, since the frost layer blocks the flow passage. In addition, the frost layer grows faster when the inlet air humidity and velocity are higher or when the air and cold wall temperatures are lower. It can be concluded that the present generalized LB model is capable of reproducing both qualitative and quantitative frosting behaviors without separate treatment of the humid air and the frost regions. The focus of this work is model development, so a number of prototype frosting problems have been simulated for validation purposes. For heating, ventilation, and air conditioning applications, it is necessary to treat complex surfaces and structures, of which the LB model is inherently capable [28]. For icing and anti-icing problems in aerospace, it is important to include turbulence effects. Existing studies $[28,48]$ have 
demonstrated that the LB method is capable of handling turbulence and complex geometries. Combining such capabilities with the present LB model would open up a wide range of applications to exploration.

\section{ACKNOWLEDGMENTS}

This work was supported by the UK Engineering and Physical Sciences Research Council under the project "UK Consortium on Mesoscale Engineering Sciences (UKCOMES)" (Grants No. EP/L00030X/1 and No. EP/R029598/1) and the China Scholarship Council (CSC, Grant No. 201706160161).

\section{APPENDIX A: THE CHAPMAN-ENSKOG ANALYSIS OF THE D2Q9 LB MODEL FOR FLOW FIELD}

In this Appendix, the Chapman-Enskog analysis is provided for the LB equation (24) to recover the hydrodynamic equations (3) and (4). To this end, the following multiscale expansions are introduced:

$$
\begin{aligned}
f_{i} & =f_{i}^{(0)}+\xi f_{i}^{(1)}+\xi^{2} f_{i}^{(2)}, \\
F_{i} & =\xi F_{i}^{(1)}+\xi^{2} F_{i}^{(2)}, \\
R_{i} & =\xi R_{i}^{(1)}+\xi^{2} R_{i}^{(2)}, \\
\partial_{t} & =\xi \partial_{t_{1}}+\xi^{2} \partial_{t_{2}}, \quad \nabla=\xi \nabla_{1},
\end{aligned}
$$

where $\xi$ is a small parameter. By applying the Taylor expansion into Eq. (24), one can obtain

$$
D_{i} f_{i}+\frac{\delta_{t}}{2} D_{i}^{2} f_{i}=-\frac{1}{\tau_{u} \delta_{t}}\left(f_{i}-f_{i}^{\mathrm{eq}}\right)+F_{i}+R_{i},
$$

where $D_{i}=\partial_{t}+\boldsymbol{e}_{i} \cdot \nabla$. Substituting Eqs. (A1a)-(A1d) into Eq. (A2) yields

$$
\begin{aligned}
\left(\partial_{t}+\right. & \left.\boldsymbol{e}_{i} \cdot \nabla\right)\left[f_{i}^{(0)}+\xi f_{i}^{(1)}+\xi^{2} f_{i}^{(2)}\right] \\
& +\frac{\delta_{t}}{2}\left(\partial_{t}+\boldsymbol{e}_{i} \cdot \nabla\right)^{2}\left[f_{i}^{(0)}+\xi f_{i}^{(1)}+\xi^{2} f_{i}^{(2)}\right] \\
= & -\frac{1}{\tau_{u} \delta_{t}}\left[f_{i}^{(0)}+\xi f_{i}^{(1)}+\xi^{2} f_{i}^{(2)}-f_{i}^{\mathrm{eq}}\right] \\
& +\xi F_{i}^{(1)}+\xi^{2} F_{i}^{(2)}+\xi R_{i}^{(1)}+\xi^{2} R_{i}^{(2)} .
\end{aligned}
$$

This gives the following set of equations at increasing orders of $\xi$ :

$$
\begin{aligned}
\xi^{0}: f_{i}^{(0)} & =f_{i}^{\mathrm{eq}}, \\
\xi^{1}: D_{1 i} f_{i}^{(0)} & =-\frac{1}{\tau_{u} \delta_{t}} f_{i}^{(1)}+F_{i}^{(1)}+R_{i}^{(1)}, \\
\xi^{2}: \partial_{t 2} f_{i}^{(0)} & +D_{1 i} f_{i}^{(1)}+\frac{\delta_{t}}{2} D_{1 i}^{2} f_{i}^{(0)}=-\frac{1}{\tau_{u} \delta_{t}} f_{i}^{(2)}+F_{i}^{(2)}+R_{i}^{(2)},
\end{aligned}
$$

where $D_{1 i}=\partial_{t_{1}}+\boldsymbol{e}_{i} \cdot \nabla_{1}$. Substituting Eq. (A4b) into Eq. (A4c) yields

$$
\begin{aligned}
\partial_{t 2} f_{i}^{(0)}+D_{1 i}\left\{\left(1-\frac{1}{2 \tau_{u}}\right) f_{i}^{(1)}+\frac{\delta_{t}}{2}\left[F_{i}^{(1)}+R_{i}^{(1)}\right]\right\} \\
=-\frac{1}{\tau_{u} \delta_{t}} f_{i}^{(2)}+F_{i}^{(2)}+R_{i}^{(2)} .
\end{aligned}
$$

With the definition of equilibrium equation $f_{i}^{\text {eq }}$ in Eq. (25), the following moments can be obtained:

$$
\begin{aligned}
\sum_{i} f_{i}^{\mathrm{eq}} & =\rho \\
\sum_{i} \boldsymbol{e}_{i} f_{i}^{\mathrm{eq}} & =\rho_{l} \boldsymbol{u} \\
\sum_{i} \boldsymbol{e}_{i} \boldsymbol{e}_{i} f_{i}^{\mathrm{eq}} & =c_{s}^{2} \rho+\frac{\rho_{l}}{\epsilon} \boldsymbol{u} \boldsymbol{u} \\
\sum_{i} \boldsymbol{e}_{i} \boldsymbol{e}_{i} \boldsymbol{e}_{i} f_{i}^{\mathrm{eq}} & =c_{s}^{2} \rho_{l}\left(\boldsymbol{u}_{\alpha} \delta_{\beta \gamma}+\boldsymbol{u}_{\beta} \delta_{\alpha \gamma}+\boldsymbol{u}_{\gamma} \delta_{\beta \alpha}\right)
\end{aligned}
$$

It is noted that based on Eq. (28) for calculating $\rho$ and $\boldsymbol{u}$, and the relationship between $f_{i}^{(0)}$ and $f_{i}^{\text {eq }}$ in Eq. (A4a), one can obtain

$$
\begin{aligned}
\sum_{i} f_{i}^{(n)} & =\frac{\delta_{t}}{2} \dot{m}^{(n)}, \text { for } n \geqslant 1, \\
\sum_{i} \boldsymbol{e}_{i} f_{i}^{(n)} & =-\frac{\delta_{t}}{2}\left[\rho_{l} F_{i}^{(n)}+Q_{i}^{(n)}\right], \quad \text { for } n \geqslant 1 .
\end{aligned}
$$

Similarly, from the definitions of source term distributions in Eqs. (26) and (27), it is derived that

$$
\begin{gathered}
\sum_{i}\left[F_{i}^{(n)}+R_{i}^{(n)}\right]=-\left(1-\frac{1}{2 \tau_{u}}\right) \dot{m}^{(n)}, \\
\sum_{i} \boldsymbol{e}_{i}\left[F_{i}^{(n)}+R_{i}^{(n)}\right]=\left(1-\frac{1}{2 \tau_{u}}\right)\left[\rho_{l} \boldsymbol{F}^{(n)}-\dot{m}^{(n)}\right], \\
\sum_{i} \boldsymbol{e}_{i} \boldsymbol{e}_{i}\left[F_{i}^{(n)}+R_{i}^{(n)}\right] \\
=\left(1-\frac{1}{2 \tau_{u}}\right)\left\{\frac{\rho_{l}}{\epsilon}\left[\boldsymbol{u} \boldsymbol{F}^{(n)}+\boldsymbol{F}^{(n)} \boldsymbol{u}\right]-c_{s}^{2} \dot{m}^{(n)}\right\} .
\end{gathered}
$$

By taking the zeroth and first lattice velocity moments of Eq. (A4b), the macroscopic equations on the $t_{1}$ timescale and $\boldsymbol{x}_{1}$ space scale are obtained,

$$
\begin{gathered}
\frac{\partial \rho}{\partial t_{1}}+\rho_{l} \nabla_{1} \cdot \boldsymbol{u}=-\dot{m}^{(1)}, \\
\rho_{l} \frac{\partial \boldsymbol{u}}{\partial t_{1}}+\nabla_{1}\left(c_{s}^{2} \rho+\frac{\rho_{l} \boldsymbol{u} \boldsymbol{u}}{\epsilon}\right)=\rho_{l} \boldsymbol{F}^{(1)}+\boldsymbol{M}^{(1)} .
\end{gathered}
$$

Then, the macroscopic equations on the $t_{2}$ timescale are derived by taking velocity moments of Eq. (A5),

$$
\begin{gathered}
\frac{\partial \rho}{\partial t_{2}}=-\dot{m}^{(2)} \\
\rho_{l} \frac{\partial \boldsymbol{u}}{\partial t_{2}}=\rho_{l} \boldsymbol{F}^{(2)}+\boldsymbol{M}^{(2)}-\nabla_{1} \cdot\left\{\left(1-\frac{1}{2 \tau_{u}}\right) \sum_{i} \boldsymbol{e}_{i} \boldsymbol{e}_{i} f_{i}^{(1)}\right. \\
\left.+\frac{\delta_{t}}{2} \sum_{i} \boldsymbol{e}_{i} \boldsymbol{e}_{i}\left[F_{i}^{(1)}+R_{i}^{(1)}\right]\right\}
\end{gathered}
$$

To proceed further, the momentum flux $\sum_{i} \boldsymbol{e}_{i} \boldsymbol{e}_{i} f_{i}^{(1)}$ needs to be evaluated. By making use of Eqs. (A4b), (A9), and (A10), 
it can be obtained that

$$
\begin{aligned}
\boldsymbol{\Pi}= & \left(1-\frac{1}{2 \tau_{u}}\right) \sum_{i} \boldsymbol{e}_{i} \boldsymbol{e}_{i} f_{i}^{(1)}+\frac{\delta_{t}}{2} \sum_{i} \boldsymbol{e}_{i} \boldsymbol{e}_{i}\left[F_{i}^{(1)}+R_{i}^{(1)}\right] \\
= & \left(\frac{1}{2}-\tau_{u}\right) \delta_{t} D_{1} i \sum_{i} \boldsymbol{e}_{i} \boldsymbol{e}_{i} f_{i}^{(0)}+\tau_{u} \delta_{t} \sum_{i} \boldsymbol{e}_{i} \boldsymbol{e}_{i}\left[F_{i}^{(1)}+R_{i}^{(1)}\right] \\
= & \left(\frac{1}{2}-\tau_{u}\right) \delta_{t}\left(\partial_{t_{1}}\left(c_{s}^{2} \rho+\frac{\rho_{l} \boldsymbol{u} \boldsymbol{u}}{\epsilon}\right)+\nabla_{1} \cdot \sum_{i} \boldsymbol{e}_{i} \boldsymbol{e}_{i} \boldsymbol{e}_{i} f_{i}^{(0)}\right. \\
& \left.-\left\{\frac{\rho_{l}}{\epsilon}\left[\boldsymbol{u} \boldsymbol{F}^{(1)}+\boldsymbol{F}^{(1)} \boldsymbol{u}\right]-c_{s}^{2} \dot{m}^{(1)}\right\}\right) \\
= & \left(\frac{1}{2}-\tau_{u}\right) c_{s}^{2} \rho_{l} \nabla_{1} \boldsymbol{u} .
\end{aligned}
$$

In the derivations, as used in Ref. [5], the terms of $O\left(M^{3}\right)$ have been neglected. Here $M=u / c_{s}$ is the Mach number. Therefore, the final form of Eq. (A12) is

$$
\rho_{l} \frac{\partial \boldsymbol{u}}{\partial t_{2}}=\rho_{l} \boldsymbol{F}^{(2)}+\boldsymbol{M}^{(2)}-\left(\frac{1}{2}-\tau_{u}\right) c_{s}^{2} \rho_{l} \nabla_{1} \cdot \nabla_{1} \boldsymbol{u} .
$$

Combining the macroscopic equations on the $t_{1}$ and $t_{2}$ scales, one can finally obtain the governing equations (3) and (4), with the $v_{e}$ given by Eq. (31).

\section{APPENDIX B: THE CHAPMAN-ENSKOG ANALYSIS OF THE D2Q9 LB MODELS FOR TEMPERATURE AND HUMIDITY FIELDS}

The Champan-Enskog technique is also deployed to recover the governing equations (5) and (6) for temperature and humidity fields. The distribution functions, time, and space are expanded as follows:

$$
\begin{aligned}
g_{i} & =g_{i}^{(0)}+\xi g_{i}^{(1)}+\xi^{2} g_{i}^{(2)}, \\
G_{i} & =\xi G_{i}^{(1)}+\xi^{2} G_{i}^{(2)}, \\
h_{i} & =h_{i}^{(0)}+\xi h_{i}^{(1)}+\xi^{2} h_{i}^{(2)},
\end{aligned}
$$

$$
\begin{aligned}
H_{i} & =\xi H_{i}^{(1)}+\xi^{2} H_{i}^{(2)}, \\
\partial_{t} & =\xi \partial_{t_{1}}+\xi^{2} \partial_{t_{2}}, \quad \nabla=\xi \nabla_{1} .
\end{aligned}
$$

Then the Taylor expansion is applied to Eqs. (32) and (38), which leads to

$$
\begin{aligned}
& D_{i} g_{i}+\frac{\delta_{t}}{2} D_{i}^{2} g_{i}=-\frac{1}{\tau_{t} \delta_{t}}\left(g_{i}-g_{i}^{\mathrm{eq}}\right)+G_{i}, \\
& D_{i} h_{i}+\frac{\delta_{t}}{2} D_{i}^{2} h_{i}=-\frac{1}{\tau_{w} \delta_{t}}\left(h_{i}-h_{i}^{\mathrm{eq}}\right)+H_{i} .
\end{aligned}
$$

Combined with Eqs. (B1a)-(B1e), the following equations in the consecutive order of $\xi$ can be obtained:

$$
\begin{gathered}
\xi^{0}: g_{i}^{(0)}=g_{i}^{\mathrm{eq}} \\
\xi^{1}: D_{1 i} g_{i}^{(0)}=-\frac{1}{\tau_{t} \delta_{t}} g_{i}^{(1)}+G_{i}^{(1)}, \\
\xi^{2}: \partial_{t 2} g_{i}^{(0)}+D_{1 i}\left[\left(1-\frac{1}{2 \tau_{t}}\right) g_{i}^{(1)}+\frac{\delta_{t}}{2} g_{i}^{(1)}\right] \\
=-\frac{1}{\tau_{t} \delta_{t}} g_{i}^{(2)}+G_{i}^{(2)}
\end{gathered}
$$

and

$$
\begin{gathered}
\xi^{0}: h_{i}^{(0)}=h_{i}^{\mathrm{eq}} \\
\xi^{1}: D_{1 i} h_{i}^{(0)}=-\frac{1}{\tau_{w} \delta_{t}} h_{i}^{(1)}+H_{i}^{(1)} \\
\xi^{2}: \partial_{t 2} h_{i}^{(0)}+D_{1 i}\left[\left(1-\frac{1}{2 \tau_{w}}\right) h_{i}^{(1)}+\frac{\delta_{t}}{2} h_{i}^{(1)}\right] \\
=-\frac{1}{\tau_{w} \delta_{t}} h_{i}^{(2)}+H_{i}^{(2)} .
\end{gathered}
$$

As conducted in Appendix A, by taking the lattice velocity moments of Eqs. (B4b) and (B4c) and Eqs. (B5b) and (B5c), the governing equations (5) and (6) can be recovered. The diffusion coefficients $\alpha_{e}$ and $D_{e}$ are given by Eqs. (37) and (41).
[1] Y. Hayashi, A. Aoki, S. Adachi, and K. Hori, J. Heat Transfer 99, 239 (1977).

[2] W. M. Yan, H. Y. Li, Y. J. Wu, J. Y. Lin, and W. R. Chang, Int. J. Heat Mass Transf. 46, 871 (2003).

[3] M. B. Bragg, D. C. Heinrich, W. O. Valarezo, and R. J. McGhee, J. Aircraft 31, 1372 (1994).

[4] C. T. Sanders, Ph.D. thesis, Delft Technical University, 1974.

[5] Z. L. Guo and T. S. Zhao, Phys. Rev. E 66, 036304 (2002).

[6] Y. X. Tao, R. W. Besant, and K. S. Rezkallah, Int. J. Heat Mass Transf. 36, 353 (1993).

[7] R. Le Gall, J. M. Grillot, and C. Jallut, Int. J. Heat Mass Transf. 40, 3177 (1997).

[8] K. A. R. Ismail and C. S. Salinas, Int. J. Refrig. 22, 425 (1999).

[9] H. Chen, R. W. Besant, and Y. X. Tao, ASHRAE Trans. 105, 252 (1999).
[10] B. Na and R. L. Webb, Int. J. Heat Mass Transf. 47, 925 (2004).

[11] A. Luer and H. Beer, Int. J. Therm. Sci. 39, 85 (2000).

[12] K. S. Lee, S. Jhee, and D. K. Yang, Int. J. Heat Mass Transf. 46, 3789 (2003).

[13] K. Lenic, A. Trp, and B. Frankovic, Appl. Therm. Eng. 29, 2534 (2009).

[14] K. Lenic, A. Trp, and B. Frankovic, Int. J. Heat Mass Transf. 52, 22 (2009).

[15] J. M. Armengol, C. T. Salinas, J. Xaman, and K. A. R. Ismail, Int. J. Therm. Sci. 104, 245 (2016).

[16] J. Cui, W. Z. Li, Y. Liu, and Y. S. Zhao, Int. J. Heat Fluid Fl. 32, 249 (2011).

[17] J. Cui, W. Z. Li, Y. Liu, and Z. Y. Jiang, Appl. Therm. Eng. 31, 447 (2011). 
[18] D. Kim, C. Kim, and K. S. Lee, Int. J. Heat Mass Transf. 82, 135 (2015).

[19] X. M. Wu, Q. Ma, F. Q. Chu, and S. Hu, Int. J. Heat Mass Transf. 96, 11 (2016).

[20] X. M. Wu, F. Q. Chu, and Q. Ma, Int. J. Heat Mass Transf. 110, 760 (2017).

[21] A. Faghri, Y. W Zhang, and J. Howell, Advanced Heat and Mass Transfer (Global Digital Press, America, 2010).

[22] S. S. Feng, J. J. Kuang, T. Wen, T. J. Lu, and K. Ichimiya, Int. J. Heat Mass Transf. 77, 1063 (2014).

[23] S. S. Feng, M. Shi. Y. F. Li, and T. J. Lu, Int. J. Heat Mass Transf. 90, 838 (2015).

[24] S. S. Feng, Y. Zhang, M. Shi, T. Wen, and T. J. Lu, Appl. Therm. Eng. 88, 315 (2015).

[25] M. Sheikholeslami, J. Mol. Liq. 265, 347 (2018).

[26] M. Sheikholeslami, J. Mol. Liq. 259, 424 (2018).

[27] D. Lycett-Brown and K. H. Luo, Phys. Rev. E 94, 053313 (2016).

[28] Q. Li, K. H. Luo, Q. J. Kang, Y. L. He, Q. Chen, and Q. Liu, Prog. Energy Combust. 52, 62 (2016).

[29] Q. Li, Q. J. Kang, M. M. Francois, Y. L. He, and K. H. Luo, Int. J. Heat Mass Transf. 85, 787 (2015).

[30] Q. Li, K. H. Luo, and X. J. Li, Phys. Rev. E 87, 053301 (2013).

[31] Z. L. Guo and T. S. Zhao, Numer. Heat Transf. B 47, 157 (2005).

[32] D. Y. Gao and Z. Q. Chen, Int. J. Therm. Sci. 50, 493 (2011).
[33] D. Y. Gao, Z. Q. Chen, D. L. Zhang, and L. H. Chen, Appl. Therm. Eng. 118, 315 (2017).

[34] Q. Liu, Y. L. He, Q. Li, and W. Q. Tao, Int. J. Heat Mass Transf. 73, 761 (2014).

[35] Q. Liu and Y. L. He, Physica A 438, 94 (2015).

[36] W. Wu, S. L. Zhang, and S. F. Wang, Int. J. Heat Mass Transf. 104, 675 (2017).

[37] S. Ergun, Chem. Eng. Prog. 48, 89 (1952).

[38] K. Vafai, J. Fluid. Mech. 147, 233 (1984).

[39] D. J. Wessel et al., ASHRAE handbook: 2001 Fundamentals (American Society of Heating, Refrigerating, and AirConditioning Engineers, Atlanta, 2001).

[40] Z. L. Guo and C. Shu, Lattice Boltzmann Method and its Applications in Engineering (World Scientific, Singapore, 2013).

[41] T. M. Lei, X. H. Meng, and Z. L. Guo, Comput. Fluids 155, 146 (2017).

[42] Y. L. He, Q. Liu, Q. Li, and W. Q. Tao, Int. J. Heat Mass Transf. 129, 160 (2019).

[43] M. N. Ozisik, Heat Conduction (Wiley, New York, 1993).

[44] C. Beckermann and R. Viskanta, Int. J. Heat Mass Transf. 31, 35 (1988).

[45] J. D. Yonko and C. F. Sepsy, ASHRAE Trans. 73, 1.1 (1967).

[46] F. Wang, C. H. Liang, and X. S. Zhang, Renew. Sust. Energ. Rev. 81, 707 (2018).

[47] W. Sheng, P. P. Liu, C. B. Dang, and G. X. Liu, Renew. Sust. Energ. Rev. 79, 806 (2017).

[48] C. K. Aidun and J. R. Clausen, Annu. Rev. Fluid Mech. 42, 439 (2010). 